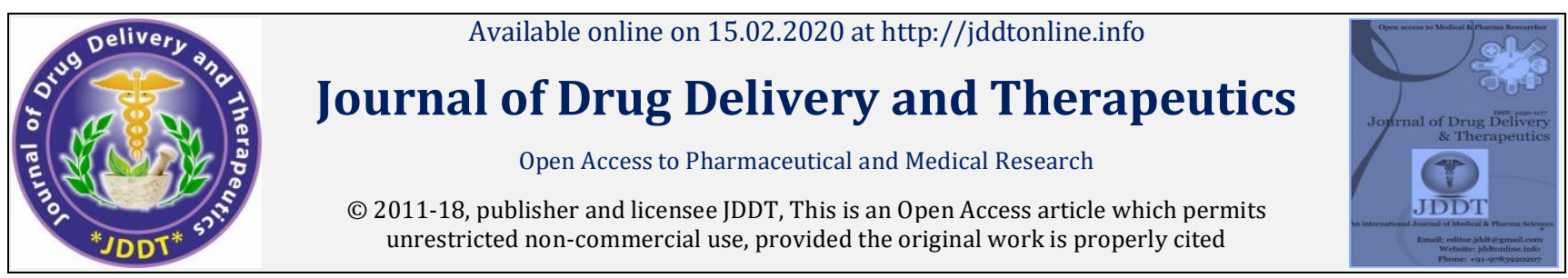

Open Access

Research Article

\title{
A Study on Appropriateness of Antibacterial Use in Acute Wards of a Teaching Hospital
}

\author{
Sanjay Jaiswal*, Sharan Shyam, Vikas Yadav \\ Armed Forces Medical College, Pune, India
}

\begin{abstract}
Objective: A study to analyse the appropriateness of use of antibacterials in acute wards of a tertiary care hospital. Design: A pharmacoepidemiological research study. Intervention: Non-interventional descriptive study. Main Outcome Measure: The antibacterial prescriptions were analyzed for their adherence to or deviation from the standard national treatment guidelines. Results: There were a total of 207 patients comprising of 35 in acute medical ward, 65 in ICU, 53 in acute surgical ward and 54 in family ward, who were prescribed antibacterials. These consisted of a total of 383 prescription counts for appropriateness of AMA use. One third of the antibacterial prescriptions of inpatients surveyed were for prophylactic use followed by the treatment of respiratory and gastrointestinal diseases. The highest prescription counts were seen with carbapenems followed by nitroimidazoles, cephalosporins, aminoglycosides and penicillins. $99 \%$ of the prescriptions were appropriate for the dose used and $49.6 \%$ of the prescriptions were inappropriate for duration of use. $74.5 \%$ of the surgical prophylaxis prescriptions were used for more than 24 hours. Ceftriaxone and cefotaxime were used instead of the recommended cefazolin, for prophylaxis. High percentage of deviation was observed with prescriptions of cefotaxime and metronidazole for empirical use. No definitive use of antimicrobials for infections was observed in the ICU. Conclusion: A high percentage of appropriateness was observed for dose of antibiotic used. The main targets identified for improvement are duration of antimicrobial use, choice of drug for surgical prophylaxis and the definitive use of antimicrobial agents.
\end{abstract}

Keywords: adherence to treatment guidelines, antibiotic audit, antimicrobials.

Article Info: Received 29 Nov 2019; Review Completed 25 Jan 2020; Accepted 04 Feb 2020; Available online 15 Feb 2020

Cite this article as:

Jaiswal S, Shyam S, Yadav V, A Study on Appropriateness of Antibacterial Use in Acute Wards of a Teaching Hospital, Journal of Drug Delivery and Therapeutics. 2020; 10(1-s):144-148 http://dx.doi.org/10.22270/jddt.v10i1-s.3927

*Address for Correspondence:

Sanjay Jaiswal, Armed Forces Medical College, Pune, India

\section{INTRODUCTION}

Wide spread and inappropriate use of antimicrobials, result in the development of multidrug resistant organisms and increase the burden of infections. This can lead to increased morbidity, mortality and length of hospital stay, adding to healthcare expenditures. The documentation of inpatients with infections is important to formulate a hospital antibiotic policy and check its adherence periodically. ${ }^{1}$ The World Health Organization (WHO) has recently revised the essential medicines list with the goal of improving rational use of antibiotics. ${ }^{2}$ However, data on the implementation of such policies especially quality indicators of antimicrobial prescriptions is lacking. ${ }^{3}$

\section{OBJECTIVE}

The present prescription analysis has been carried out in departments of a tertiary care teaching hospital, expected to have the highest burden of infectious diseases, in order to bring out the appropriateness of use of anti-bacterials.

\section{MATERIAL AND METHODS}

The study was conducted in four wards, namely acute medical, acute surgical, intensive care unit (ICU) and family wards of a teaching hospital of western India, authorized a total of 1082 beds. In each of the four wards, the antibiotic treatment of infectious diseases was analyzed by follow up of inpatients, those who were prescribed antimicrobial agents (AMA), for a calendar month each in May, June, July and August 2018 respectively. Data recorded included the demographic data like age and sex, the clinical diagnosis, antibiotics administered to the patient during their stay in the hospital, the duration of antibiotic use, the duration of hospital stay, and the result of their treatment as cured/discharged/transferred out. The prescriptions were analyzed for appropriateness by checking their adherence to or deviation from the standard national guidelines. From the collective data of antibiotics prescribed in all the four acute wards, the top five antibiotics consumed from each acute ward were chosen to assess for appropriateness of use. 
The dose and duration of antibiotic use were assessed by analyzing adherence to the "Treatment Guidelines for Antimicrobial Use in Common Syndromes', published by the Indian Council of Medical Research in 2017.4

\section{RESULTS}

Inpatients of acute medical ward, ICU, acute surgical ward and family wards, those who were prescribed AMA, were followed up for a calendar month each. There were a total of 207 patients comprising of 35 in acute medical ward, 65 in ICU, 53 in acute surgical ward and 54 in family ward, who were prescribed AMA and on longitudinal study. These consisted of a total of 383 prescription counts for appropriateness of AMA use. The demographics of inpatients prescribed AMA during the survey is depicted in Table1.

Table1: Demographic data of patients on antimicrobial agents $(n=207)$

\begin{tabular}{|cc|}
\hline Age (years) & Percentage of patients (\%) \\
$<18$ & 3 \\
$18-30$ & 8 \\
$31-60$ & 50 \\
$>60$ & 39 \\
Sex & Percentage of patients (\%) \\
Male & 65 \\
Female & 35 \\
\hline
\end{tabular}

The pattern of systemic infections encountered during the study is shown in Fig1.
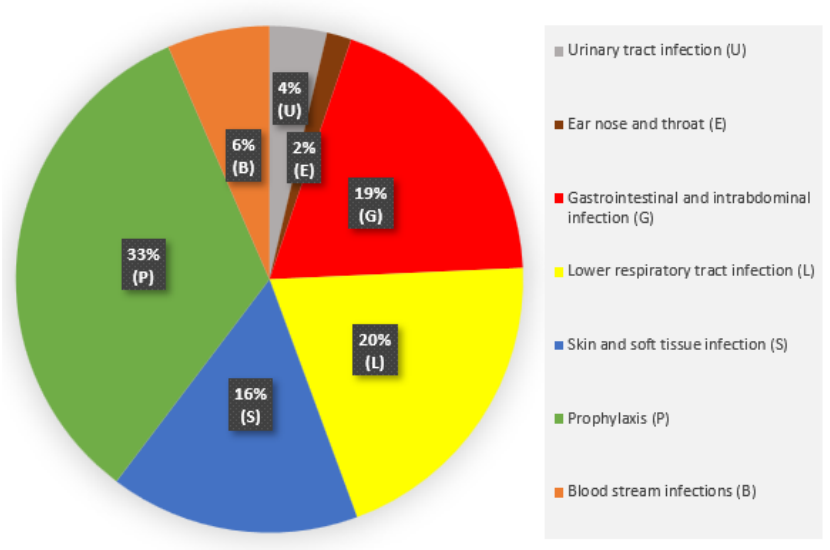

Figure 1: System wise distribution of AMA prescriptions $(n=383)$

One third of the antibacterial prescriptions of inpatients surveyed were for prophylactic use followed by the treatment of respiratory and gastrointestinal diseases. The consumption of AMA in each acute ward of the hospital during the month is shown in Fig2. The highest prescription counts were seen with carbapenems followed by nitroimidazoles, cephalosporins, aminoglycosides and penicillins.

Ten AMA were selected for appropriateness study based on the top five AMA consumed in each ward. The details of appropriateness based on purpose of use, adherence to treatment guidelines and duration of AMA is depicted in Tables 2,3 and 4 .
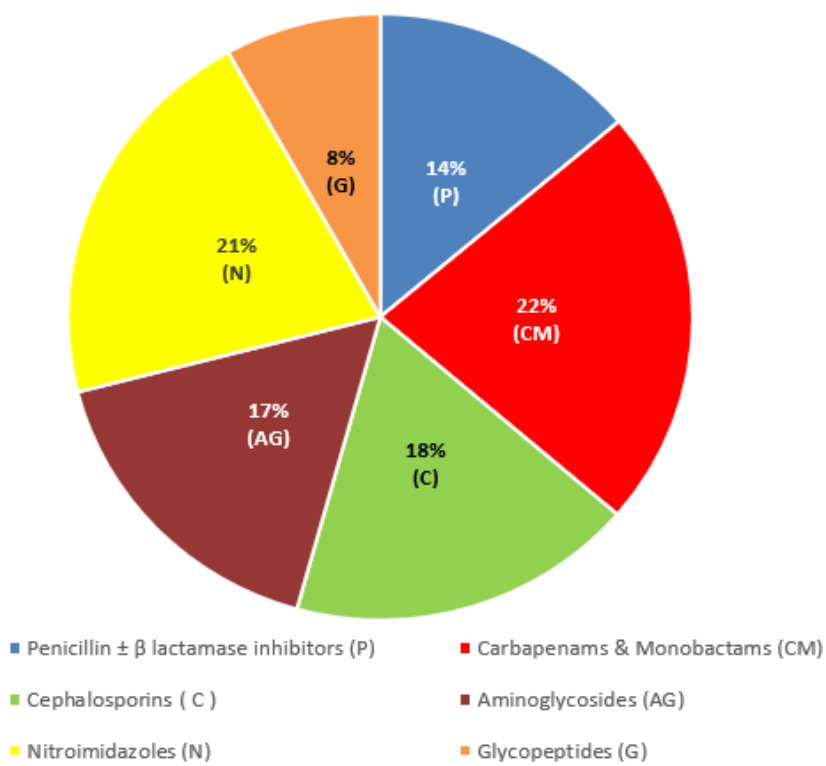

Figure 2: Class wise distribution of AMA prescriptions $(n=383)$

Note- Percentages in the above figure represent prescription counts of AMA

Table 2: Appropriateness of AMA for purpose of use

\begin{tabular}{|c|c|c|c|c|}
\hline \multirow[b]{2}{*}{ Name of antibiotic } & \multicolumn{4}{|c|}{ Use (P/TE/TD) (\%) } \\
\hline & AM & AS & $\mathrm{ICU}$ & FAM \\
\hline \multirow[b]{2}{*}{ Amikacin } & & $P(50)$ & $P(50)$ & $\mathrm{P}(78)$ \\
\hline & TE (100) & TE (50) & TE (50) & TE (22) \\
\hline \multirow[b]{2}{*}{ Amoxicillin+Clavulanic acid } & & $P(44)$ & $\mathrm{P}(33)$ & $P(25)$ \\
\hline & TE (100) & $\mathrm{TE}(56)$ & $\mathrm{TE}(67)$ & TE (75) \\
\hline \multirow[b]{3}{*}{ Cefotaxime } & & & & $P(65)$ \\
\hline & & $P(74)$ & $P(60)$ & TE (30) \\
\hline & TE (100) & $\operatorname{TE}(26)$ & $\operatorname{TE}(40)$ & $\mathrm{TD}(5)$ \\
\hline \multirow[b]{2}{*}{ Cefuroxime } & & $P(50)$ & & \\
\hline & NIL & $\operatorname{TE}(50)$ & NIL & NIL \\
\hline \multirow[b]{2}{*}{ Ceftriaxone } & TE (92) & & $P(25)$ & TE (89) \\
\hline & $\mathrm{P}(8)$ & $\operatorname{TE}(100)$ & TE (75) & $\mathrm{P}(11)$ \\
\hline \multirow[b]{3}{*}{ Imipenem } & & $P(8)$ & & \\
\hline & & TE (84) & & TE (57) \\
\hline & TE (100) & TD (8) & $\operatorname{TE}(100)$ & $\mathrm{P}(43)$ \\
\hline \multirow[b]{2}{*}{ Meropenem } & TE (83) & & $P(10.5)$ & \\
\hline & TD (17) & $\operatorname{TE}(100)$ & TE (89.5) & TE (100) \\
\hline \multirow[b]{2}{*}{ Metronidazole } & & $P(48)$ & $P(50)$ & TE (61) \\
\hline & TE (100) & TE (52) & TE (50) & $\mathrm{P}(39)$ \\
\hline Piperacillin+Tazobactam & $\operatorname{TE}(100)$ & TE (100) & $\operatorname{TE}(100)$ & TE (100) \\
\hline Teicoplanin & TE (100) & TE (100) & TE $(100)$ & TE (100) \\
\hline
\end{tabular}

Legend

$\mathrm{AM}=$ acute medical ward (males)

$\mathrm{AS}=$ acute surgical ward (males)

ICU $=$ intensive care units

$\mathrm{FAM}=$ family ward (females)

$\mathrm{P}=$ prophylactic

$\mathrm{TE}=$ therapeutic empirical

$\mathrm{TD}=$ therapeutic definitive

Highlighted in green are prescriptions with AMA use for definitive therapy 
Table 3: Appropriateness of AMA as per standard treatment guidelines

\begin{tabular}{|c|c|c|c|c|}
\hline \multirow[b]{2}{*}{ Name of antibiotic } & \multicolumn{4}{|c|}{ Guideline (A /D+ /D-) (\%) } \\
\hline & $\mathrm{AM}$ & AS & $\mathrm{ICU}$ & FAM \\
\hline \multirow[b]{3}{*}{ Amikacin } & & & $A(50)$ & \\
\hline & & & D- (43) & $A(83)$ \\
\hline & $\mathrm{A}(100)$ & D- $(100)$ & $\mathrm{D}+(7)$ & D- (17) \\
\hline \multirow[b]{3}{*}{ Amoxicillin+Clavulanic acid } & & $A(22)$ & & \\
\hline & & D- $(67)$ & $A(22)$ & $A(25)$ \\
\hline & $A(100)$ & $\mathrm{D}+(11)$ & D- $(78)$ & D- (75) \\
\hline \multirow[b]{2}{*}{ Cefotaxime } & $A(80)$ & & & \\
\hline & D- $(20)$ & D- $(100)$ & D- $(100)$ & D- $(100$ \\
\hline \multirow[b]{2}{*}{ Cefuroxime } & & $A(50)$ & & \\
\hline & NIL & D- $(50)$ & NIL & NIL \\
\hline \multirow[b]{2}{*}{ Ceftriaxone } & $A(83)$ & & $A(75)$ & $A(56)$ \\
\hline & D- (17) & $\mathrm{A}(100)$ & D- (25) & D- (44) \\
\hline \multirow[b]{2}{*}{ Imipenem } & & $A(62)$ & $A(93)$ & $A(14)$ \\
\hline & $\mathrm{A}(100)$ & D- $(38)$ & D- (7) & D- (86) \\
\hline \multirow[b]{3}{*}{ Meropenem } & $A(66)$ & & & \\
\hline & D- (17) & & $A(89)$ & $A(50)$ \\
\hline & $\mathrm{D}+(17)$ & $A(100)$ & D- (11) & D- (50) \\
\hline \multirow[b]{2}{*}{ Metronidazole } & & $A(14)$ & $A(50)$ & $A(28)$ \\
\hline & D- $(100)$ & D- $(86)$ & D- $(50)$ & D- $(72)$ \\
\hline \multirow[b]{2}{*}{ Piperacillin+Tazobactam } & & & $A(94)$ & $A(80)$ \\
\hline & $A(100)$ & $A(100)$ & $D-(6)$ & D- (20) \\
\hline \multirow[b]{3}{*}{ Teicoplanin } & $\mathrm{A}(33.3)$ & & $A(76)$ & $A(40)$ \\
\hline & D- $(33.3)$ & $A(17)$ & D- $(6)$ & D- (40) \\
\hline & $D+(33.3)$ & D- $(83)$ & $D+(18)$ & $D+(20)$ \\
\hline
\end{tabular}

\section{Legend}

AM = acute medical ward (males)

AS = acute surgical ward (males)

ICU = intensive care units

$\mathrm{FAM}=$ family ward (females)

$\mathrm{A}=$ adherent to guideline

$\mathrm{D}+=$ deviation from guideline with justification

D- = deviation from guideline without justification

Highlighted in green are prescriptions with deviation from guideline with justification.

Highlighted in red are prescriptions with $>60 \%$ deviation without justification

Table 4: Appropriateness of AMA as per duration of use

\begin{tabular}{l|c|c|c|c|}
\multirow{2}{*}{ Name of antibiotic } & \multicolumn{4}{|c|}{ Duration-Yes (Y)/No (N) } \\
\cline { 2 - 5 } & AM & AS & ICU & FAM \\
\hline Amikacin & $\mathrm{Y}(100)$ & $\mathrm{Y}(43)$ & $\mathrm{Y}(36)$ & $\mathrm{Y}(17)$ \\
\hline Amoxicillin+Clavulanic acid & $\mathrm{Y}(100)$ & $\mathrm{Y}(22)$ & $\mathrm{Y}(22)$ & $\mathrm{Y}(25)$ \\
\hline Cefotaxime & $\mathrm{Y}(50)$ & $\mathrm{Y}(53)$ & $\mathrm{Y}(40)$ & $\mathrm{Y}(41)$ \\
\hline Cefuroxime & $\mathrm{NIL}$ & $\mathrm{Y}(100)$ & $\mathrm{NIL}$ & $\mathrm{NIL}$ \\
\hline Ceftriaxone & $\mathrm{Y}(83)$ & $\mathrm{Y}(100)$ & $\mathrm{Y}(44)$ & $\mathrm{Y}(67)$ \\
\hline Imipenem & $\mathrm{Y}(67)$ & $\mathrm{Y}(54)$ & $\mathrm{Y}(71)$ & $\mathrm{Y}(43)$ \\
\hline Meropenem & $\mathrm{Y}(100)$ & $\mathrm{N}(100)$ & $\mathrm{Y}(58)$ & $\mathrm{Y}(25)$ \\
\hline Metronidazole & $\mathrm{Y}(25)$ & $\mathrm{Y}(45)$ & $\mathrm{Y}(31)$ & $\mathrm{Y}(28)$ \\
\hline Piperacillin+Tazobactam & $\mathrm{Y}(100)$ & $\mathrm{Y}(100)$ & $\mathrm{Y}(59)$ & $\mathrm{Y}(40)$ \\
\hline Teicoplanin & $\mathrm{Y}(33)$ & $\mathrm{Y}(33)$ & $\mathrm{Y}(71)$ & $\mathrm{Y}(40)$ \\
\hline
\end{tabular}

\section{Legend}

AM $=$ acute medical ward (males)

AS = acute surgical ward (males)

$\mathrm{ICU}=$ intensive care units

$\mathrm{FAM}=$ family ward (females)

$\mathrm{Y}=\mathrm{Yes} \mathrm{N}=\mathrm{No}$

Highlighted in red are prescriptions with $<40 \%$ appropriate duration of use.
Out of the 383 prescriptions analyzed from all the wards, only one prescription did not mention a clear indication for the use of the antibiotic (0.026\%). 99\% of the doses used in the prescriptions, showed adherence to the treatment guidelines. $100 \%$ of the prescriptions analyzed showed no likelihood of drug-drug interaction. Only $50.4 \%$ of the prescriptions were appropriate for duration of use. Inappropriate duration of use of AMA was highest in the family ward (64\%), followed by the ICU (52\%).

$78.3 \%$ of the antibiotic use was empirical, with only $0.8 \%$ antibiotics started for definitive treatment, after sensitivity results. Most of the empirical drugs were continued due to the improvement in clinical condition of the patient, denying the need to change the antibiotic. 55.4\% of the prescriptions were in accordance with the antibiotics recommended for the condition by the national treatment guidelines. $3 \%$ were deviating from the guidelines but with justification for their choice of antibiotic. $41.6 \%$ prescriptions were deviating from the national guidelines, without any justification (mostly with surgical prophylaxis).

$3 \%$ of the prescriptions justified the deviation from guidelines due to reasons like poor clinical response or due to antibiogram report. $100 \%$ adherence to guidelines was seen with the use of amikacin, amoxicillin+clavulanic acid, imipenem and piperacillin+tazobactam in the acute medical ward. Similarly, the use of ceftriaxone, meropenem and piperacillin+tazobactam showed $100 \%$ adherence in the surgical ward. The highest use of antibiotics for prophylactic purpose was in the family ward (29\%), followed closely by the acute surgical ward $(27.4 \%)$ and the intensive care units (19\%).

\section{DISCUSSION}

This epidemiological study on use of AMA was carried out in a Government teaching hospital that provides free treatment to its entitled patients. The case sheets of inpatients and other medical records are meticulously maintained in the concerned office. In this study, we explored the appropriateness of antimicrobial use in terms of indication, dose, duration of use and adherence to national recommendations.

Of the total 383 prescriptions analyzed for appropriateness in our study, $99.97 \%$ prescriptions had an indication for use mentioned, which is higher than the studies reported in India and abroad.5-8 Even though the dose used was adherent to the national treatment guidelines in $99 \%$ of the prescriptions, $49.6 \%$ of the prescriptions were inappropriate for duration of use. In some cases, AMA had to be changed due to non-availability in the hospital pharmacy. The high percentage of inappropriate duration of use is mostly attributable to the use of antibiotics for more than 24 hours in surgical prophylaxis cases. $30.8 \%$ of the prescriptions surveyed in the acute wards were for surgical prophylaxis and $74.5 \%$ of the AMAs were used for more than 24 hours. This finding is less than the reports of other workers. ${ }^{9}$ Nevertheless, it is quite high compared to data from some developed nations where 9\% of AMA use was prophylactic and $40 \%$ was used for more than 24 hours. 7,8

The ICU and family ward had the highest proportion of inappropriate use of AMA based on drug of choice for the indication and the duration of use. The postoperative cases are admitted to the ICU before stepping down to the respective wards. As per guidelines, all cases of surgical prophylaxis need to be used for less than 24 hours. ${ }^{10}$ Ceftriaxone and cefotaxime of WATCH group were used instead of the recommended agent, cefazolin, which is an Access group AMA of WHO EML. ${ }^{2}$ In many cases, 
metronidazole was administered even though an anerobic infection was not anticipated. Use of two AMAs with overlapping anti-bacterial spectrum of action was also observed in the study.

Cefotaxime use was found to be deviating from standard treatment guidelines and without justification in 3 out of four wards. This was mostly due to preference of cefotaxime over cefazolin as surgical prophylactic agent. The use of metronidazole showed high deviation in adherence to treatment guidelines, in the acute wards either due to its prophylactic use in Class I/ II surgical wounds or in combination with ceftriaxone in certain systemic infections.

Use of antibiotics for definitive treatment was highest in the acute medical ward (2\%), followed by acute surgical (1\%) and family ward $(0.5 \%)$. There was no definitive treatment antibiotic use in the ICU. This could be because of life threatening infections suspected at the time of admission in the ICU and AMA had mostly been started before the culture and sensitivity results were available. The use of AMA for definitive therapy of infections has been found to be very low in our study.

The use of antibiograms can improve appropriateness of AMA prescribing. A survey amongst medical residents in the United States revealed that only $12 \%$ of respondents identified antibiograms as a resource when prescribing empiric antibiotic therapy. ${ }^{11}$

An observational study in Netherlands has highlighted the shorter length of hospital stay due to appropriate antibiotic use in admitted patients. ${ }^{12}$ In another Dutch study, $29.3 \%$ of the prescriptions were deemed inappropriate mainly due to unjustified indication. ${ }^{13} \mathrm{~A}$ study at an Australian hospital reported that $47 \%$ of the antibiotic use was found to be deviating from the national guidelines or microbiological results. ${ }^{14}$

An Indian study showed that $93.3 \%$ of the antibiotic use in ICU was for therapeutic use and $89.3 \%$ antimicrobial prescriptions were adherent to the hospital antibiotic policy. ${ }^{15} \mathrm{~A}$ prospective observational study found antibiotic use longer than the recommended duration (33.3\%) and use in non-infectious conditions (32.4\%). ${ }^{16}$ In yet another study, $64.1 \%$ of prescriptions were appropriate which increased to $77.4 \%$ after a 12 month follow up. ${ }^{5}$

A Netherlands study reported $37.4 \%$ of infection prescriptions inappropriate, especially with quinolones and amoxicillin+clavulanic acid. 17

A study in Australian hospitals on AMA use reported 52.4\% of the prescriptions as appropriate. ${ }^{7}$ This was higher when AMAs were used for empirical treatment (80.4\%) compared to use in prophylaxis (40.6\%). Inappropriateness was often due to incorrect selection or due to prophylactic use exceeding 24 hours (40\%). A study on antibiotic prescription pattern in northern India has highlighted the lapses in rational use of anti-bacterials primarily due to inadequate training.18 Our study found a higher appropriateness on choice of AMA and similar empirical use as above but for a longer duration of use for surgical prophylaxis.

This study has several strengths. Each of the acute wards in the study was followed up for a month to pick up prescriptions containing AMA, unlike other studies where point prevalence studies were conducted for investigating appropriateness. This ensures that the results of our study are more representative of the status in these wards. Day specific procedures are not missed out since all days in a week were covered.
Limitations are that some departments like pediatrics, orthopedics and gynecology have been excluded from the study. Further studies including these departments are required for results more representative of the hospital.

\section{CONCLUSION}

Methods to de-escalate the AMA use among patients would encourage early hospital discharge and reduce the burden of antimicirobial resistance. Polypharmacy and empirical use of high- end AMA need to be curbed at once. The national guidelines must be made more accessible to the clinicians and awareness regarding the same needs to improve. The existing antibiotic policy of the hospital needs to be modified especially for surgical prophylaxis, and duration of therapy. Periodic release of regional antibiotic susceptibility data and education on updated national treatment guidelines of infections are important steps in promoting rational use of AMA in health care institutions. To summarize, it is perceived that the antimicrobial stewardship programme of the hospital needs more attention and strict implementation since empirical use of AMA is very high.

\section{CONFLICT OF INTEREST}

Nil

\section{REFERENCES}

1. Malcolm W, Nathwani D, Davey P, Cromwell T, Patton A, Reilly J, et al. From intermittent antibiotic point prevalence surveys to quality improvement: experience in Scottish hospitals. AntimicrobResist Infect Control. 2013; 2(1):3.

2. World Health Organization Model List of Essential Medicines, 21st List, 2019. Geneva: World Health Organization; 2019. Licence: CC BY-NC-SA 3.0 IGO.

3. Holloway KA, Rosella L, Henry D. The impact of WHO essential medicines policies on inappropriate use of antibiotics. PLoS One. 2016 Mar 22; 11(3):e0152020.

4. Indian Council of Medical Research (ICMR). Treatment Guidelines for Antimicrobial Use in Common Syndromes. ICMR, Department of Health Research, New Delhi, India; 2017. [cited 15 May 2019]Available from: https://www.icmr.nic.in/sites/default/files/guidelines/treatme nt_guidelines_for_antimicrobial.pdf.

5. Sikkens JJ, Van Agtmael MA, Peters EJ, Lettinga KD, Van Der Kuip M, Vandenbroucke-Grauls CM, et al. Behavioral approach to appropriate antimicrobial prescribing in hospitals: the Dutch Unique Method for Antimicrobial Stewardship (DUMAS) participatory intervention study. JAMA Intern Med. 2017; 177(8):1130-8.

6. Ansari F, Erntell M, Goossens H, Davey P, ESAC II Hospital Care Study Group. The European surveillance of antimicrobial consumption (ESAC) point-prevalence survey of antibacterial use in 20 European hospitals in 2006. Clin Infect Dis. 2009; 49(10):1496-504.

7. Cotta MO, Robertson MS, Upjohn LM, Marshall C, Liew D, Buising KL. Using periodic point-prevalence surveys to assess appropriateness of antimicrobial prescribing in Australian private hospitals. Intern Med J. 2014; 44(3):240-6.

8. James R, Upjohn L, Cotta M, Luu S, Marshall C, Buising K, Thursky K. Measuring antimicrobial prescribing quality in Australian hospitals: development and evaluation of a national antimicrobial prescribing survey tool. J Antimicrob Chemother 2015; 70(6):1912-8.

9. Pathak A, Mahadik K, Dhaneria SP, Sharma A, Eriksson B, Lundborg CS. Surveillance of antibiotic consumption using the "focus of infection" approach in 2 hospitals in Ujjain, India. PLoS One. 2012; 7(6):e38641.

10. Ansari F, Erntell M, Goossens H, Davey P, ESAC II Hospital Care Study Group. The European surveillance of antimicrobial consumption (ESAC) point-prevalence survey of antibacterial use in 20 European hospitals in 2006. Clin Infect Dis. 2009; 49(10):1496-504.

11. Tallman GB, Vilches-Tran RA, Elman MR, Bearden DT, Taylor JE, Gorman PN, et al. Empiric antibiotic prescribing decisions 
among medical residents: the role of the antibiogram. Infection Control \&Hospital Epidemiology. 2018; 39(5):578-83.

12. van den Bosch CM, Geerlings SE, Natsch S, Prins JM, Hulscher ME. Quality indicators to measure appropriate antibiotic use in hospitalized adults. Clinical Infectious Diseases. 2014; 60(2):281-91.

13. Akhloufi H, Streefkerk RH, Melles DC, de Steenwinkel JE, Schurink CA, Verkooijen RP, van der Hoeven CP, Verbon A. Point prevalence of appropriate antimicrobial therapy in a Dutch university hospital. Eur JClin Microbiol Infect Dis. 2015; 34(8):1631-7.

14. Ingram PR, Seet JM, Budgeon CA, Murray R. Point-prevalence study of inappropriate antibiotic use at a tertiary Australian hospital. Intern Med J. 2012; 42(6):719-21.

15. Shah N, Joshi A, Ganguly B. Impact of antibiotic stewardship program on prescribing pattern of antimicrobials in patients of medical intensive care unit. Journal of clinical and diagnostic research: JClin Diagn Res. 2017; 11(7):FC11-FC15.

16. Hecker MT, Aron DC, Patel NP, Lehmann MK, Donskey CJ. Unnecessary use of antimicrobials in hospitalized patients: current patterns of misuse with an emphasis on the antianaerobic spectrum of activity. Arch Intern Med. 2003; 163(8):972-8.

17. Willemsen I, Groenhuijzen A, Bogaers D, Stuurman A, van Keulen P, Kluytmans J. Appropriateness of antimicrobial therapy measured by repeated prevalence surveys. Antimicrob Agents Chemother. 2007; 51(3):864-7.

18. Kaur A, Bhagat R, Kaur N, Shafiq N, Gautam V, Malhotra S, Suri V, Bhalla A. A study of antibiotic prescription pattern in patients referred to tertiary care center in Northern India. Ther Adv Infect Dis. 2018; 5(4):63-8. 